http://dx.doi.org/10.32911/as.2017.v10.n2.165

Aporte Santiaguino. 10 (2), 2017: 225-232

ISSN 2070-836X

\title{
Evaluación comparativa de ocho variedades de papa (Solanum tuberosum) en el centro poblado de Uchuyacu distrito y provincia de Huaraz departamento de Áncash 2016-2017
}

Eighth comparison evaluation of eight potato varieties (Solanum tuberosum) in the center populated of Uchuyacu district and province of Huaraz department of Ancash 2016-2017

José Ramírez Maldonado ${ }^{1}$, Teofanes Mejía Anaya ${ }^{1}$ y Carlos Fernández López ${ }^{1}$

\section{RESUMEN}

El objetivo fue comprobar el mejor rendimiento de ocho variedades de papa en una determinada altitud y a condiciones ecológicas sobre los 2.800 m.s.n.m. El diseño experimental utilizado ha sido el diseño de bloque completamente al azar, la instalación del cultivo se realizó el 5 de noviembre de 2016, se cosechó el 28 de mayo de 2017. Las variedades utilizadas fueron: Yungay, Amarilis, Huayro, Canchan, Perricholi, Milagros, Marina y Kori-INIA. Se realizó un abonamiento a razón de $\mathrm{N}=180 \mathrm{~kg} \mathrm{~g} / \mathrm{ha}$., $\mathrm{P}=$ $180 \mathrm{~kg} / \mathrm{ha}$. y K $=120 \mathrm{~kg} / \mathrm{ha}$ mezclado con $3 \mathrm{Tm} / \mathrm{ha}$ de guano de corral de vacuno. Las variedades que destacaron con un óptimo rendimiento de acuerdo a la prueba de comprobación de medias de Tukey, con diferencias estadísticamente significativas al 1 $\%$, fueron: la variedad Perricholi con $32.020 \mathrm{Tm} /$ ha promedio de rendimiento, seguido por Kori INIA con 27.979 Tm/ha el menor rendimiento lo tiene la variedad Huayro con solamente 19.374 Tm/ha. La información de estos resultados permitirá a los agricultores adoptar decisiones acertadas evitando riesgos de pérdidas durante la siembra de este cultivo.

Palabras clave: variedad; rendimiento; abonamiento.

\section{ABSTRACT}

The objective of the research was verify the best performance of eight potato varieties at a certain altitude and ecological conditions over 2.800 m.a.s.l. The experimental

1 Universidad Nacional Santiago Antúnez de Mayolo. Huaraz, Perú. 
design used was the completely randomized block design, the crop installation was performed on november 5, 2016, harvested on May 28. 2017. The varieties used were: Yungay, Amarilis, Huayro, Canchan, Perricholi, Milagros, Marina and Kori-INIA. A fertilization rate of $\mathrm{N}=180 \mathrm{~kg} g / \mathrm{ha}, \mathrm{P}=180 \mathrm{~kg} / \mathrm{ha}$ and $\mathrm{K}=120 \mathrm{~kg} / \mathrm{ha}$. was mixed with $3 \mathrm{Tm} /$ ha of cattle barnyard guano. The varieties that stood out with an optimum performance according to the test of means test of Tukey, with differences statistically significant to $1 \%$, were: the variety Perricholi with $32.020 \mathrm{Tm} /$ ha average of yield, followed by Kori INIA with $27.979 \mathrm{Tm} /$ ha has the lowest yield is the Huayro variety with only $19.374 \mathrm{Tm} / \mathrm{ha}$. The information of these results will allow the farmers to make wise decisions avoiding risks of losses during the sowing of this crop.

Keywords: variety; performance; fertilization.

\section{INTRODUCCIÓN}

En la producción agrícola de la región Áncash, el cultivo de la papa constituye uno de los mayores cultivos atractivos en su programa de inversión para el agricultor, obviamente debido a la gran demanda, al alto consumo y a las bondades alimenticias que tiene este tubérculo tales como proteína 4,6\%, carbohidratos 30,53\%, fibra cruda 3,48 \%, ácidos orgánicos 1,0\%, sólidos totales 36,8 \%, grasa 0,20 \% C- 54 mg/100 gr y minerales entre otros nutrientes (MINAG-DGPA, 2002), de ahí se desprende su relevancia por ser uno de los alimentos más consumidos por la población.

Sin embargo, existe la necesidad de establecer qué variedades demuestran óptimos rendimientos en un determinado agro ecosistema, toda vez que los rendimientos actuales en nuestro medio es alrededor de 9,2 Tm/ha (MINAG, DGPA, 2002). Es por estas consideraciones que se ha realizado el trabajo de investigación cuyos objetivos específicos fueron los siguientes: a) Instalar ocho (08) variedades de papa en el centro poblado de Uchuyacu ubicado en el distrito y provincia de Huaraz; b) Evaluar los rendimientos por cada uno de las variedades de papa en estudio; c) Promover la producción de la variedad(es) de papa de óptimo rendimiento para su cultivo en zonas análogas en la que se ha realizado el estudio.

Durante la siembra y cultivo de las diferentes variedades de papa se logró comprobar que difieren en su capacidad productiva, características de los tubérculos, época de maduración, resistencia a insectos, enfermedades y en cualidades culinarias. Así una determinada variedad es superior en ciertas zonas y marcadamente inferior en otras, es decir, de acuerdo a las características propias de un agro ecosistema donde se desarrolla el cultivo de la papa. Por consiguiente, los agricultores deben solicitar el asesoramiento a entidades académicas como las universidades o algún otro centro experimental agrícola sobre el rendimiento y adaptabilidad de una o más variedades de acuerdo a la zona de cultivo (Richard, 1989). 


\section{MATERIALES Y MÉTODOS}

Durante el estudio se evaluaron ocho variedades de papa provenientes del centro poblado de Macashca - centro poblado perteneciente al distrito de Huaraz asentado en una altura de 3.355 m s.n.m. Estas variedades son las siguientes: Yungay, Amarilis, Huayro, Canchan, Perricholi, Milagros, Marina y Kori-INIA. El ensayo se desarrolló en finca de agricultores del centro poblado de Uchuyacu en un clima de tipo frío a templado, en una altitud de 3.300 m s.n.m. Para el abonamiento se utilizó la dosis de 180-180 y $120 \mathrm{~kg} /$ ha de N, P y K respectivamente más 3 Tm/ha de guano de corral de vacuno. Se realizaron 2 aporques entre los 46 y 65 días después de la siembra, debido a que no existió incidencia significativa de plagas y enfermedades. Como medida preventiva se aplicaron pesticidas contra áfidos, gusanos de tierra y la Phytophthora infestans, (produjo una enfermedad conocida como tizón tardío muy levemente). Se aplicaron 4 riegos por gravedad con una frecuencia de 35 días, los riegos restantes fueron complementados por las lluvias. Se trabajó con el Diseño de Bloque Completamente al Azar usando 08 variedades y 5 repeticiones donde el área neta del experimento para los fines de estimación de rendimiento fue de $384 \mathrm{~m} 2$ los distanciamientos fueron de $0,80 \mathrm{~m}$ entre surco y $0.30 \mathrm{~m}$ entre planta, cada sub parcela del bloque tiene $6 \mathrm{~m}$ de largo por 1,6 $\mathrm{m}$ de ancho ( 2 surcos centrales) donde 1,6 $\mathrm{m} \times 6 \mathrm{~m}=9,6 \mathrm{~m} \times 40 \mathrm{~T}=384 \mathrm{~m} 2$ entre $0,24 \mathrm{~m} 2 /$ planta $=1.600$ plantas entre 8 variedades $=200$ plantas evaluadas por cada variedad. El cultivo tuvo un desarrollo normal durante su período vegetativo, se tuvo un ligero ataque del insecto conocido como Diabrotica speciosa (es una especie de insecto coleóptero de la familia Chrysomelidae) pero luego se controló eficazmente con la aplicación de un licuado de ajo a razón de 1/2 kilo por 10 litros de agua más $1 / 2$ barra de jabón Bolívar y la presencia de controladores biológicos como Opius sp.

\section{RESULTADOS}

Tabla 1. ANVA del rendimiento total de 8 variedades de papa

\begin{tabular}{lccccc}
\hline \multicolumn{1}{c}{ F.V. } & G.L. & S.C. & C.M. & Fc & SIGNIFICACIÓN \\
\hline BLOQUES & 4 & 9,48 & 2,37 & 1,22 & NS \\
TRATAMIENTOS & 7 & 545,50 & 77,93 & 40,17 & $* *$ \\
ERROR EXPER. & 28 & 54,36 & 1,94 & & --- \\
\hline \multicolumn{1}{c}{ TOTAL } & 39 & 609,34 & --- & --- & --- \\
\hline
\end{tabular}

C.V. $=6,04 \%$ 
Tabla 1.1. Prueba de Tukey en el rendimiento total de 8 variedades de papa en TM/HA

\begin{tabular}{clccc}
\hline ORD. MERI. & VARIEDADES & TM/HA & SIGNIF. & $\%$ \\
\hline 1 & PERRICHOLI & 32,020 & $\mathrm{a}$ & \\
2 & KORI-INIA & 27,979 & $\mathrm{ab}$ & \\
3 & MARIVA & 25,416 & $\mathrm{bc}$ & \\
4 & CANCHAN & 23,124 & $\mathrm{~cd}$ & \multirow{2}{*}{$\%$} \\
5 & MILAGROS & 22,916 & $\mathrm{~cd}$ & \\
6 & AMARILIS & 20,624 & $\mathrm{~cd}$ & \\
7 & YUNGAY & 20,416 & $\mathrm{~d}$ & \\
8 & HUAYRO & 19,374 & $\mathrm{~d}$ & \\
\hline
\end{tabular}

En el ANVA realizado para el rendimiento total de papa, no existe diferencias significativas entre los bloques, existe diferencia estadística altamente significativa entre las variedades de papa en estudio, para la prueba de comprobación de medias de Tukey existe diferencias estadísticamente significativas al 1\%, donde la mayor performance lo tiene la variedad Perricholi con $32.020 \mathrm{Tm} /$ ha promedio, seguido por Kori INIA con 27.979 Tm/ha. El menor rendimiento lo tiene la variedad Huayro con $19.374 \mathrm{Tm} / \mathrm{ha}$. probablemente este bajo rendimiento se deba a que es una variedad triploide siendo las restantes tetraploides. En las siguientes tablas 1 y 1.1 se aprecian los rendimientos totales de cada variedad.

Tabla 2. ANVA para el rendimiento de papa de primera calidad

\begin{tabular}{lccccc}
\hline \multicolumn{1}{c}{ F.V. } & G.L. & S.C. & C.M. & Fc & SIGNIFICACIÓN \\
\hline BLOQUES & 4 & 0,796 & 0,199 & 0,2145 & NS \\
TRATAMIENTOS & 7 & 88,743 & 12,677 & 13,66 & $* *$ \\
ERROR EXPER. & 28 & 25,972 & 0,9276 & -- & --- \\
\hline \multicolumn{1}{c}{ TOTAL } & 39 & 115,972 & --- & --- & --- \\
\hline \multicolumn{7}{c}{ C.V. $=12,10 \%$}
\end{tabular}

Tabla 2.1. Prueba de Tukey para el rendimiento promedio de papa de primera calidad en $9,6 \mathrm{~m}^{2}$

\begin{tabular}{clccc}
\hline $\begin{array}{c}\text { ORD. } \\
\text { MERI. }\end{array}$ & VARIEDADES & $\begin{array}{c}\text { PESO } \\
\text { EN Kg/9.6 } \\
\mathbf{m}^{\mathbf{2}}\end{array}$ & SIGNIF. & $\%$ \\
\hline $\mathbf{1}$ & PERRICHOLI & 11,40 & $\mathrm{a}$ & \\
$\mathbf{2}$ & KORI-INIA & 8,72 & $\mathrm{~b}$ & \\
$\mathbf{3}$ & MARIVA & 8,00 & $\mathrm{~b}$ & \\
$\mathbf{4}$ & MILAGROS & 8,00 & $\mathrm{~b}$ & $1 \%$ \\
$\mathbf{5}$ & CANCHAN & 7,60 & $\mathrm{~B}$ & \\
$\mathbf{6}$ & AMARILIS & 7,00 & $\mathrm{~B}$ & \\
$\mathbf{7}$ & YUNGAY & 6,60 & $\mathrm{~B}$ & \\
$\mathbf{8}$ & HUAYRO & 6,40 & $\mathrm{~B}$ & \\
\hline
\end{tabular}


Tabla 2.2. Prueba de tukey para el rendimiento promedio de papa de primera calidad en HA

\begin{tabular}{clccc}
\hline $\begin{array}{c}\text { ORD. } \\
\text { MERI. }\end{array}$ & \multirow{2}{*}{ VARIEDADES } & $\begin{array}{c}\text { PESO } \\
\overline{\boldsymbol{X}}_{\text {EN }} \text { TM/HA }\end{array}$ & SIGNIF. & \multirow{2}{*}{$\%$} \\
\hline $\mathbf{1}$ & PERRICHOLI & 11,875 & $\mathrm{a}$ & \\
$\mathbf{2}$ & KORI-INIA & 9,083 & $\mathrm{~b}$ & \\
$\mathbf{3}$ & MARIVA & 8,333 & $\mathrm{~B}$ & \\
$\mathbf{4}$ & MILAGROS & 8,333 & $\mathrm{~B}$ & \multirow{1}{*}{$\%$} \\
$\mathbf{5}$ & CANCHAN & 7,916 & $\mathrm{~B}$ & \\
$\mathbf{6}$ & AMARILIS & 7,291 & $\mathrm{~b}$ & \\
$\mathbf{7}$ & YUNGAY & 6,875 & $\mathrm{~b}$ & \\
$\mathbf{8}$ & HUAYRO & 6,666 & $\mathrm{~b}$ & \\
\hline
\end{tabular}

En el ANVA realizado para el rendimiento de papa de primera como se aprecia en la tabla 2 y 2.1, que se explica a continuación, se encontró alta significación entre los tratamientos para la calidad primera de papa siendo el coeficiente de variabilidad de $12,1 \%$, la variedad que destaca en la prueba de medias es la Perricholi en las demás variedades no se encuentra diferencia estadística.

Tabla 3. ANVA para el rendimiento de papa de segunda calidad

\begin{tabular}{lccccc}
\hline \multicolumn{1}{c}{ F.V. } & G.L. & S.C. & C.M. & Fc & SIGNIFICACIÓN \\
\hline BLOQUES & 4 & 8,320 & 2,08 & 1,71 & NS \\
TRATAMIENTOS & 7 & 35,478 & 5,068 & 4,17 & $* *$ \\
ERROR EXPER. & 28 & 34,032 & 1,215 & -- & --- \\
\hline \multicolumn{1}{c}{ TOTAL } & 39 & 77,830 & --- & --- & --- \\
\hline & C.V. $=13,34 \%$ &
\end{tabular}

Tabla 3.1. Prueba de Tukey para rendimiento promedio de papa de segunda calidad en 9,6 $\mathrm{m}^{2}$ $\mathrm{P}=0,05$

\begin{tabular}{clccc}
\hline $\begin{array}{c}\text { ORD. } \\
\text { MERI. }\end{array}$ & VARIEDADES & $\begin{array}{c}\text { PESO } \\
\text { EN Kg/9.6 }\end{array}$ & SIGNIF. & $\%$ \\
\hline $\mathbf{\mathbf { m } ^ { 2 }}$ & & \\
\hline $\mathbf{1}$ & PERRICHOLI & 9,74 & $\mathrm{a}$ & \\
$\mathbf{2}$ & KORI-INIA & 9,40 & $\mathrm{ab}$ & \\
$\mathbf{3}$ & MARIVA & 9,20 & $\mathrm{ab}$ & \\
$\mathbf{4}$ & AMARILIS & 8,00 & $\mathrm{ab}$ & $5 \%$ \\
$\mathbf{5}$ & YUNGAY & 7,60 & $\mathrm{ab}$ & \\
$\mathbf{6}$ & MILAGROS & 7,40 & $\mathrm{~b}$ & \\
$\mathbf{7}$ & CANCHAN & 7,40 & $\mathrm{~b}$ & \\
$\mathbf{8}$ & HUAYRO & 7,40 & $\mathrm{~b}$ & \\
\hline
\end{tabular}


Tabla 3.2. Prueba de Tukey para rendimiento promedio de papa de segunda calidad en HA $\mathrm{P}=0,05$

\begin{tabular}{clccc}
\hline $\begin{array}{c}\text { ORD. } \\
\text { MERI. }\end{array}$ & \multirow{2}{*}{ VARIEDADES } & $\begin{array}{c}\text { PESO } \\
\boldsymbol{X}_{\text {EN TM } / \text { HA }}\end{array}$ & SIGNIF. & \multirow{2}{*}{$\%$} \\
\hline $\mathbf{1}$ & PERRICHOLI & 10,145 & $\mathrm{a}$ & \\
$\mathbf{2}$ & KORI-INIA & 9,792 & $\mathrm{ab}$ & \\
$\mathbf{3}$ & MARIVA & 9,583 & $\mathrm{ab}$ & \\
$\mathbf{4}$ & AMARILIS & 8,333 & $\mathrm{ab}$ & $5 \%$ \\
$\mathbf{5}$ & YUNGAY & 7,916 & $\mathrm{ab}$ & \\
$\mathbf{6}$ & MILAGROS & 7,708 & $\mathrm{~b}$ & \\
$\mathbf{7}$ & CANCHAN & 7,708 & $\mathrm{~b}$ & \\
$\mathbf{8}$ & HUAYRO & 7,708 & $\mathrm{~b}$ & \\
\hline
\end{tabular}

En el ANVA para papa de segunda calidad existen diferencias altamente significativa entre los tratamientos con un coeficiente de variabilidad de $13,34 \%$, en la prueba de medias de tukey no existe diferencias significativas entre el primero y la quinta variedad, las variedades de menor performance son Canchán, Huayro y Milagros. Como se ofreció en los siguientes tablas $3,3.1$ y 3.2 .

Tabla 4. ANVA para el rendimiento de papa de tercera calidad

\begin{tabular}{lccccc}
\hline \multicolumn{1}{c}{ F.V. } & G.L. & S.C. & C.M. & Fc & SIGNIFICACIÓN \\
BLOQUES & 4 & 4,78 & 11,950 & 0,96 & NS \\
TRATAMIENTOS & 7 & 105,81 & 151,157 & 12,24 & $* *$ \\
ERROR EXPER. & 28 & 34,57 & 12,346 & -- & --- \\
\hline \multicolumn{1}{c}{ TOTAL } & 39 & 145,16 & --- & --- & --- \\
\hline & C.V. $=16,30 \%$ &
\end{tabular}

Tabla 4.1. Prueba de Tukey para rendimiento promedio de papa de tercera calidad en 9,6 $\mathrm{m}^{2}$

\begin{tabular}{clccc}
\hline \multirow{2}{*}{$\begin{array}{c}\text { ORD. } \\
\text { MERI. }\end{array}$} & \multirow{2}{*}{ VARIEDADES } & $\begin{array}{c}\text { PESO } \\
\text { EN } \\
\mathbf{K g} / \mathbf{9 . 6} \mathbf{~ m}^{\mathbf{2}}\end{array}$ & SIGNIF. & $\%$ \\
\hline $\mathbf{1}$ & PERRICHOLI & 9,60 & $\mathrm{a}$ & \\
$\mathbf{2}$ & KORI-INIA & 8,74 & $\mathrm{ab}$ & \\
$\mathbf{3}$ & MARIVA & 7,20 & $\mathrm{abc}$ & \\
$\mathbf{4}$ & CANCHAN & 7,20 & $\mathrm{abc}$ & \multirow{2}{*}{$\%$} \\
$\mathbf{5}$ & MILAGROS & 6,60 & $\mathrm{bc}$ & \\
$\mathbf{6}$ & YUNGAY & 5,40 & $\mathrm{c}$ & \\
$\mathbf{7}$ & HUAYRO & 5,00 & $\mathrm{c}$ & \\
$\mathbf{8}$ & AMARILIS & 4,80 & $\mathrm{c}$ & \\
\hline
\end{tabular}


Tabla 4.2. Prueba de Tukey para rendimiento promedio de papa de tercera calidad en HA

\begin{tabular}{clccc}
\hline ORD. & \multirow{4}{*}{ VARIEDADES } & $\begin{array}{c}\text { PESO } \\
\text { EN } \\
\text { MERI. }\end{array}$ & SIGNIF. & $\%$ \\
\hline $\mathbf{1}$ & PERRICHOLI & 10,000 & $\mathrm{a}$ & \\
$\mathbf{2}$ & KORI-INIA & 9,104 & $\mathrm{ab}$ & \\
$\mathbf{3}$ & MARIVA & 7,500 & $\mathrm{abc}$ & \\
$\mathbf{4}$ & CANCHAN & 7,500 & $\mathrm{abc}$ & \multirow{1}{*}{$\%$} \\
$\mathbf{5}$ & MILAGROS & 6,875 & $\mathrm{bc}$ & \\
$\mathbf{6}$ & YUNGAY & 5,625 & $\mathrm{c}$ & \\
$\mathbf{7}$ & HUAYRO & 5,208 & $\mathrm{c}$ & \\
$\mathbf{8}$ & AMARILIS & 5,000 & $\mathrm{c}$ & \\
\hline
\end{tabular}

En el ANVA para papa de tercera calidad existe alta significación entre los tratamientos con un coeficiente de variabilidad de $16,3 \%$, realizada la prueba de Tukey no existe diferencias entre las variedades de 1 al 4 siendo las de menor performance Huayro y Amarilis, como se aprecia en las tablas 4, 4.1 y 4.2.

\section{DISCUSIÓN}

El rendimiento de la variedad Perricholi sobresale debido a la producción mayor de primera calidad con $11.875 \mathrm{Tm} / \mathrm{ha}$, en la segunda calidad con $10.145 \mathrm{Tm} /$ ha y en la tercera calidad con $10.000 \mathrm{Tm} / \mathrm{ha}$, teniendo un rendimiento total de $32.020 \mathrm{Tm} / \mathrm{ha}$. Según PROSAAMER (2008), En el estudio de rentabilidad de la papa en la campaña agrícola 2003-2004 en el Valle del Tambo, indica que bajo esas condiciones climáticas se obtuvo un rendimiento promedio de $29.516 \mathrm{Tm} /$ ha de la variedad Perricholi.

En segundo lugar, la variedad Kori-INIA tuvo un rendimiento total de $27.979 \mathrm{Tm} /$ ha, cabe destacar que estas dos variedades antes mencionadas son sobresalientes con una producción superior a $25 \mathrm{Tm} / \mathrm{ha}$, la cual indica que son variedades que se deben promocionar para su cultivo. SEINPA (1994) indica con relación a la fertilización del suelo durante el cultivo de papa, al igual que otros cultivos, absorbe del suelo todos los minerales necesarios, estos minerales en el suelo suman 14 los elementos requeridos los mismos que son: carbono, oxígeno, hidrógeno, nitrógeno, fósforo y potasio como elementos mayores, y entre los micro nutrientes azufre, magnesio, hierro, manganeso, boro, zinc, cobre y molibdeno. El experimento se condujo con una fertilización mezclada de fertilizante sintético con abono orgánico de corral de ovino y vacuno, con esta atingencia según Óscar (2005) existen razones que resaltan el carácter fertilizante de los estiércoles. Una de ellas es que incluyen todos los nutrientes vegetales, pues, además de los tres esenciales, también contienen magnesio, calcio, azufre y micronutrientes. También, hay que señalar que una parte del nitrógeno contenido en estos residuos se encuentra en forma directamente disponible para las plantas. Por último, se deberá tener en cuenta que una porción de los nutrientes (particularmente en el caso del nitrógeno, del fósforo y de los microelementos que se halla en los estiércoles pasarán a formar parte del humus, quedando almacenados disponible en el suelo. 
La variedad Huayro tuvo un rendimiento menor con relación a las otras variedades con un total de $19.374 \mathrm{Tm} / \mathrm{h}$, probablemente este bajo rendimiento se deba a que es una variedad triploide siendo las restantes tetraploides. Según INIA (2009), en su publicación "Caracterización morfológica y agronómica de 61 variedades nativas de papa", indica que la variedad Huayro tiene un rendimiento relativo de 25-30 Tm/ha, la cual no coincide con la investigación realizada en este caso. Es probable que esta relación dependa de las características físico químicas del suelo que requiere este tubérculo.

\section{CONCLUSIONES}

Las variedades que mejor se comportaron en el rendimiento total es Perricholi y KoriINIA, en la que sobresale nítidamente la variedad Perricholi por su producción de papa de primera calidad con un rendimiento total de $32.020 \mathrm{Tm} /$ ha y seguida de KoriINIA con un rendimiento total de $27,979 \mathrm{Tm} / \mathrm{ha}$, cabe destacar que estas variedades sobresalientes están con una producción superior a $25 \mathrm{Tm} /$ ha, la cual indica que son variedades que se deben promocionar para su cultivo.

Teniendo en cuenta también que la variedad Huayro tuvo un rendimiento menor que las otras variedades con un total de 19,374 Tm/ha, probablemente esté bajo rendimiento se deba a que es una variedad triploide siendo las restantes tetraploides.

\section{REFERENCIAS BIBLIOGRÁFICAS}

INIA (Instituto Nacional de Innovación Agraria). 2009. Caracterización morfológica y agronómica de 61 variedades nativas de papa. Programa Nacional de Investigación en Papa. Perú.

Sosa, Óscar. 2005. «Cátedra de Manejo de Tierras». Agromensajes, Argentina: Facultad de ciencias Agrarias de la Universidad Nacional de Rosario. 09

MINAG-DGPA. 2002. Diagnóstico y perspectivas del desarrollo de la papa.

PROSAAMER (Programa de Servicio de Apoyo para Acceder a los Mercados Rurales). 2008. Boletín del estudio de Rentabilidad "La papa". Ministerio de Agricultura. Lima-Perú.

Richard, D. 1989. Producción agrícola de diversas especies de cultivos. Lima - Perú. 276-292.

SEINPA .1994. Semilla e Investigación de papa. Perteneciente a la Oficina de Información Agraria (OIA). Lima, Perú. Catálogo de semilla básica de papa en el Perú.

Recibido: $13 / 07 / 17$

Aceptado: 26/10/17

\section{Correspondencia}

José Ramírez Maldonado

joseram_7@hotmail.com 